\title{
Detection performance and inversion processing of logging-while-drilling extra-deep azimuthal resistivity measurements
}

\author{
Lei Wang $^{1,2,3} \cdot$ Shao-Gui Deng ${ }^{1,2,3} \cdot$ Pan Zhang ${ }^{1,2,3} \cdot$ Ying-Chang Cao ${ }^{1} \cdot$ Yi-Ren Fan ${ }^{1,2,3} \cdot$ Xi-Yong Yuan $^{1,2,3}$
}

Received: 9 November 2018 / Published online: 14 October 2019

(c) The Author(s) 2019

\begin{abstract}
We present systematic investigations on the physics, detection performance and inversion of logging-while-drilling extradeep azimuthal resistivity measurements (EDARM). First, the definitions of EDRAM measurements are discussed, followed by the derivation of the attenuation and phase-shift geometrical factors to illustrate the relative contributions of formation units to the observed signals. Then, a new definition of detection depth, which considers the uncertainty of inversion results caused by the data noise, is proposed to quantify the detection capability of EDARM. Finally, the Bayesian theory associated with Markov chain Monte Carlo sampling is introduced for fast processing of EDARM data. Numerical results show that EDARM is capable of detecting the azimuth and distance of remote bed boundaries, and the detection capability increases with increasing spacing and resistivity contrast. The EDARM tool can accommodate a large range of formation resistivity and is able to provide the resistivity anisotropy at arbitrary relative dipping angles. In addition, multiple bed boundaries and reservoir images near the borehole are readily obtained by using the Bayesian inversion.
\end{abstract}

Keywords Extra-deep azimuthal resistivity measurements (EDARM) · Detection performance · Inversion method · Reservoir imaging $\cdot$ Detection of multiple bed boundaries

\section{Introduction}

Geosteering is one of the key techniques for the exploration and development of complex oil/gas reservoirs ( $\mathrm{Li}$ et al. 2005; Wei et al. 2010; Bittar and Aki 2015). The kernel of this technique lies in the capability of detecting remote geological geometries which can be up to several meters away from the borehole (Wang et al. 2016; Yang et al. 2016; Wu et al. 2017). Two types of electromagnetic (EM) logging tools, i.e., conventional resistivity measurement and

\section{Edited by Jie Hao}

Shao-Gui Deng

dengshg@upc.edu.cn

1 School of Geosciences, China University of Petroleum, Qingdao 266580, Shandong, China

2 Laboratory for Marine Mineral Resources, Qingdao National Laboratory for Marine Science and Technology, Qingdao 266071, Shandong, China

3 CNPC Key Laboratory for Well Logging, China University of Petroleum, Qingdao 266580, Shandong, China azimuthal resistivity measurement (ARM), have been specially designed for this purpose. The former, equipped with a coaxial transmitter and coaxial receiver, is capable of providing accurate formation resistivity (Hagiwara 1996). However, the non-azimuthal property of conventional resistivity measurement has restricted its further geosteering application. By contrast, ARM incorporating a transverse or tilted antenna has eliminated the boundary azimuth uncertainty and enables the detection of a bed boundary up to $5 \mathrm{~m}$ away (Li and Wang 2016; Bittar et al. 2009; Wang et al. 2018b). Despite these advantages, the depth of detection (DoD) of current EM tools is still relatively shallow. It is necessary to improve the deep and azimuthal detection capability of EM measurements to bridge the gap between the limited DoD of logging methods and the low resolution of seismic imaging.

The first commercial extra-deep azimuthal resistivity measurement (EDARM), Geosphere, was introduced by Schlumberger (Dupuis and Denichou 2015). The new technique, which has a maximum DoD up to tens of meters, is able to detect multiple bed boundaries and to image the geological structures in real time (Seydoux et al. 2014). Compared with previous EM logging measurement, EDARM has 
the following different aspects: (1) modularized transmitter $(\mathrm{T})$ and receiver $(\mathrm{R})$ which allows for flexible T-R spacing; (2) relative lower operating frequency ranging from $1 \mathrm{kHz}$ to $100 \mathrm{kHz}$; (3) measurements of tensor magnetic fields by using orthogonal T/R antennae (Hong and Yang 2011; Gao et al. 2013). To date, very little research has been directed to EDARM and the physics, detection performance and sensitivity of EDARM remain unclear (Chaumont-Frelet et al. 2018; Li et al. 2018; Zeng et al. 2018). Systematic study is required to quantify the classical response and data processing methods of EDARM to provide accurate and reliable nearby boundaries and formation structure.
EDARM uses modularized T/R antennae (see Fig. 1) and the flexible spacing can range from 5 to $35 \mathrm{~m}$. In addition, the operating frequency also decreases. Taking Geosphere for example, the operating frequencies are $2 \mathrm{kHz}, 6 \mathrm{kHz}, 12 \mathrm{kHz}, 48 \mathrm{kHz}$ and $96 \mathrm{kHz}$, respectively. Due to the large DoD of EDARM, multiple boundaries may contribute to the tool's response. To obtain the bed boundary, anisotropic resistivity and relative dip angle simultaneously, EDARM measures the magnetic tensor, which includes $x x, y y, z z, z x$ and $x z$ components.

To magnify the sensitivity to specific formation parameters, EDARM combines different components and delivers four types of measurements which are defined as follows:

$$
\begin{aligned}
& \text { Mode } 1:\left\{\mathrm{USDA}=20 \log _{10}\left|\frac{V_{z z}-V_{z x}}{V_{z z}+V_{z x}} \cdot \frac{V_{z z}+V_{x z}}{V_{z z}-V_{x z}}\right|, \quad \mathrm{USDP}=-\operatorname{angle}\left(\frac{V_{z z}-V_{z x}}{V_{z z}+V_{z x}} \cdot \frac{V_{z z}+V_{x z}}{V_{z z}-V_{x z}}\right)\right\} \\
& \text { Mode } 2:\left\{\mathrm{UADA}=20 \log _{10}\left|\frac{V_{z z}-V_{z x}}{V_{z z}+V_{z x}} \cdot \frac{V_{z z}-V_{x z}}{V_{z z}+V_{x z}}\right|, \quad \mathrm{UADP}=-\operatorname{angle}\left(\frac{V_{z z}-V_{z x}}{V_{z z}+V_{z x}} \cdot \frac{V_{z z}-V_{x z}}{V_{z z}+V_{x z}}\right)\right\} \\
& \text { Mode } 3:\left\{\mathrm{UHRA}=20 \log _{10}\left|2 V_{z z} /\left(V_{x x}+V_{y y}\right)\right|, \quad \mathrm{UHRP}=-\operatorname{angle}\left(2 V_{z z} /\left(V_{x x}+V_{y y}\right)\right)\right\} \\
& \text { Mode } 4:\left\{\mathrm{UHAA}=20 \log _{10}\left|V_{x x} / V_{y y}\right|, \quad \mathrm{UHAP}=-\operatorname{angle}\left(V_{x x} / V_{y y}\right)\right\}
\end{aligned}
$$

In this paper, the EDARM physics, detection performance and data processing methods are investigated thoroughly. In the first section, the definitions of different EDARM modes are discussed. In the second section, a new definition is proposed to quantify the DoD of EDARM. Next, the sensitivities of EDARM to formation parameters (i.e., boundary, anisotropy and relative dip) are investigated. In the last section, the stochastic Bayesian method associated Markov Chain Monte Carlo (MCMC) sampling is introduced to invert the EDARM data and to image the formation structure.

\section{Physics of extra-deep azimuthal resistivity measurement (EDARM)}

Generally, the tool's DoD is mainly dependent on the T-R spacing. The larger the spacing, the deeper the DoD is. However, increasing spacing may result in overlong tool length. Therefore, where $V_{i j}$ depicts the voltage of $j$-oriented receiver irradiated by the $i$-oriented transmitter. USDA and USDP depict the Ultra-deep Symmetrized Directional Attenuation and Ultradeep Symmetrized Directional Phase-shift measurements, respectively. UADA and UADP signify the Ultra-deep Antisymmetrized Directional Attenuation and Ultra-deep Antisymmetrized Directional Phase-shift measurements. UHRA and UHRP are the Ultra-deep Harmonic Resistivity Attenuation and Ultra-deep Harmonic Resistivity Phaseshift measurements. UHAA and UHAP correspond to the Ultra-deep Harmonic Anisotropy Attenuation and Ultradeep Harmonic Anisotropy Phase-shift measurements.

Mode 1 and Mode 2 are the boundary detection mode and dipping-detection mode, respectively. They use the same coaxial and cross-coupling components. The difference between the two modes is that the former uses a symmetric configuration to maximize the sensitivity to bed boundaries
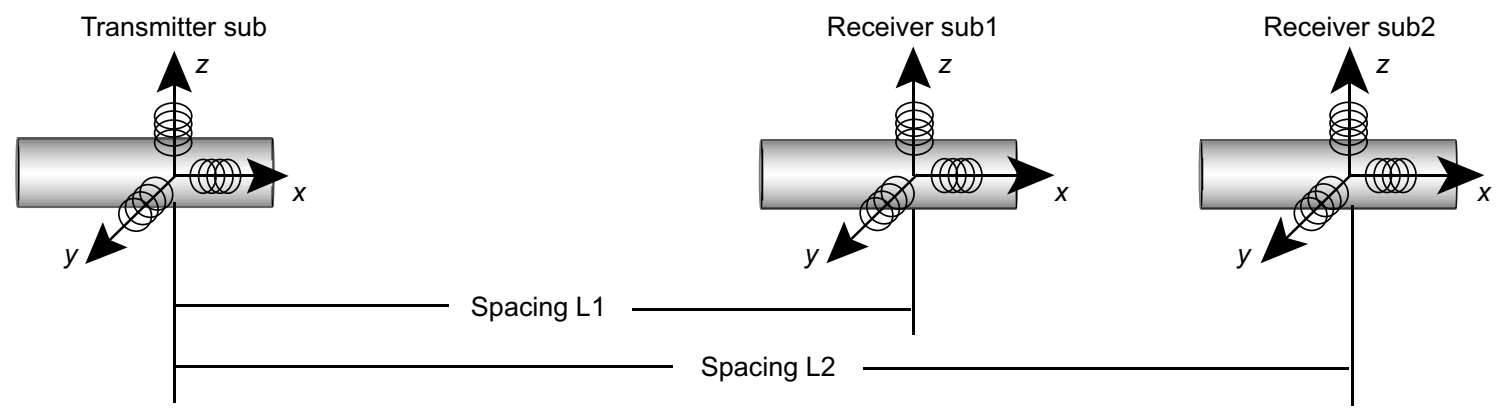

Fig. 1 Tool configuration of EDARM 
whereas the latter adopts an antisymmetric configuration to eliminate the boundary effect and to obtain the resistivity anisotropy and relative dip angle. In Mode 3, the $x x$ and $z z$ components are combined to measure the formation resistivity. Mode 4 is also designed to quantify the resistivity anisotropy by using the difference between $x x$ and $y y$ components. It should be noted that the output of the aforementioned definition is attenuation and phase shift rather than the voltage signals. This type of output not only alleviates the effect of electronic shift to measured signals, but also normalizes the signals for different spacing and frequencies.

\section{Detection performance of EDARM}

\subsection{Geometrical factor}

As a powerful tool for the analysis of the detection performance of induction logging measurements, the geometrical factor (GF) is capable of providing the relative contributions of formation units to measured signals (Wang et al. 2015). However, current GF theory is only available for the voltage signal and is not applicable to the attenuation and phase-shift signals. To address this problem, we extend the GF theory to EDARM and the detailed derivation is given in "Appendix." Assuming the transmitter and receiver lie in $\mathbf{r}_{T}\left(0,0,-\frac{L}{2}\right)$ and $\mathbf{r}_{R}\left(0,0, \frac{L}{2}\right)$ in cylindrical system $(\rho, \varphi, z)$, the attenuation GF (g) of Mode 1-Mode 4 are expressed as follows: where $r_{R}=\sqrt{\rho^{2}+(z-L / 2)^{2}}$ and $r_{T}=\sqrt{\rho^{2}+(z+L / 2)^{2}}$. $k$ is the wave number, $k^{2}=i \omega \mu \sigma . \mu$ is the magnetic permeability of free space. $\omega$ and $\sigma$ are the angular frequency and formation conductivity, respectively.

When the operating frequency is $12 \mathrm{kHz}$ and the formation resistivity is $20.0 \Omega \mathrm{m}$, the isosurfaces of EDARM GF are presented in Fig. 2, where the transmitter and receiver are at $(0,0,-7.5)$ and $(0,0,7.5)$, respectively. The red and blue colors are the surfaces of constant responses at the $\pm 90 \%$ response points. For a fixed mode, the attenuation GF shows a similar pattern to the phase-shift mode. The isosurface of UHRA is axially symmetric with respect to the z-axis, which indicates the non-azimuthal property of the formation resistivity measurement. For the first two modes, the contribution of formation units beside $x o z$ plane to the response is amplitude equal but with the opposite sign. The difference between the two modes is that the former is symmetric with respect to $z=0$ plane, whereas the latter is antisymmetric. If the resistivity of the beds besides $y=0$ plane is different, the UADA response is always zero, while the value of USDA depends on the resistivity contrast. For the geometrical factor of Mode 4, the positive and negative contributions show interphase distribution. Despite the azimuthal sensitivity of UHAA, its overall measured signal is zeros in a homogeneous isotropic medium due to the cancelation of the opposite contributions.

$$
\begin{aligned}
& g_{\mathrm{USDA}}=\operatorname{real}\left\{\frac{\frac{L^{2} \rho^{5}}{2 r_{R}^{6} r_{T}^{6}}[\cos \varphi(z+L / 2)-\sin \varphi(z-L / 2)]}{\left[\frac{1}{4 \pi L^{3}} e^{i k L}(-2 i k L+2)\right]^{2}}\left[\left(1-i k r_{R}\right)\left(1-i k r_{T}\right) e^{i k\left(r_{R}+r_{T}\right)}\right]^{2}\right\} \\
& g_{\mathrm{UADA}}=\operatorname{real}\left\{\frac{\frac{L^{2} \rho^{5}}{2 r_{R}^{6} r_{T}^{6}}[\cos \varphi(z+L / 2)+\sin \varphi(z-L / 2)]}{\left[\frac{1}{4 \pi L^{3}} e^{i k L}(-2 i k L+2)\right]^{2}}\left[\left(1-i k r_{R}\right)\left(1-i k r_{T}\right) e^{i k\left(r_{R}+r_{T}\right)}\right]^{2}\right\} \\
& g_{\mathrm{UHRA}}=\operatorname{real}\left\{\frac{\pi L^{4} \rho}{\left.r_{R}^{3} r_{T}^{3} e^{i k L}\left[\frac{\rho^{2}}{-i k L+1}-\frac{\rho^{2}+2(z+L / 2)(z-L / 2)}{k^{2} L^{2}+i k L-1}\right]\left(1-i k r_{R}\right)\left(1-i k_{b} r_{T}\right) e^{i k\left(r_{R}+r_{T}\right)}\right\}}\right. \\
& g_{\mathrm{UHAA}}=\operatorname{real}\left\{\frac{2 \pi L^{4} \rho^{3}\left(\sin ^{2} \varphi-\cos ^{2} \varphi\right)}{r_{R}^{3} r_{T}^{3} e^{i k L}\left(k^{2} L^{2}+i k L-1\right)}\left(1-i k r_{R}\right)\left(1-i k_{b} r_{T}\right) e^{i k\left(r_{R}+r_{T}\right)}\right\}
\end{aligned}
$$



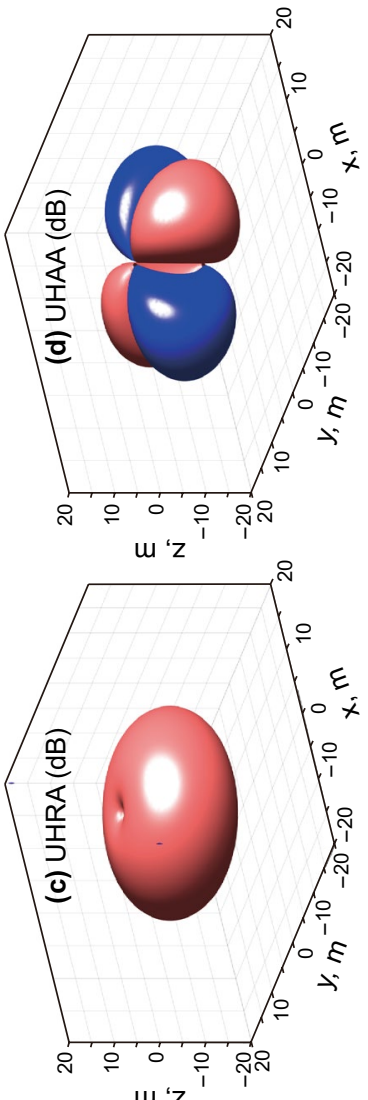

m'z
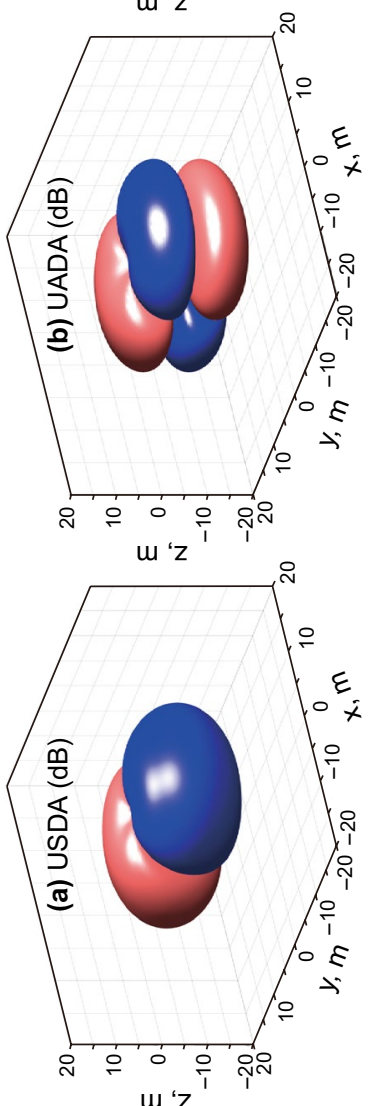

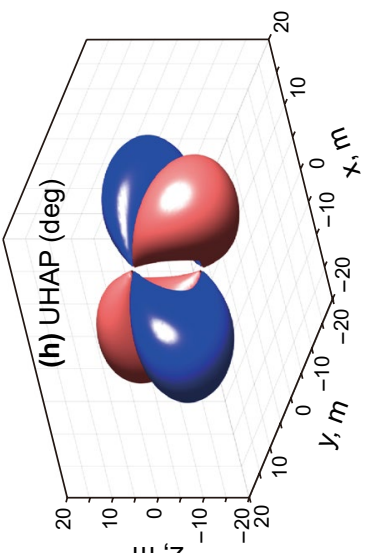

u' $\mathrm{z}$

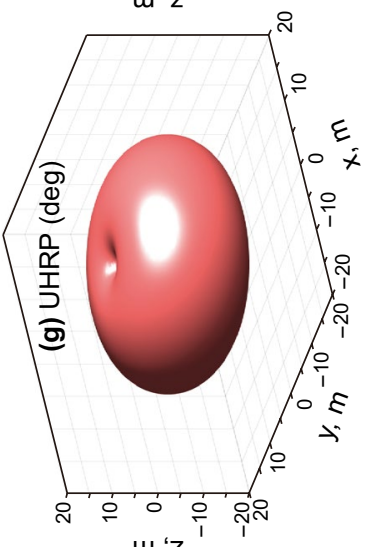

w'z

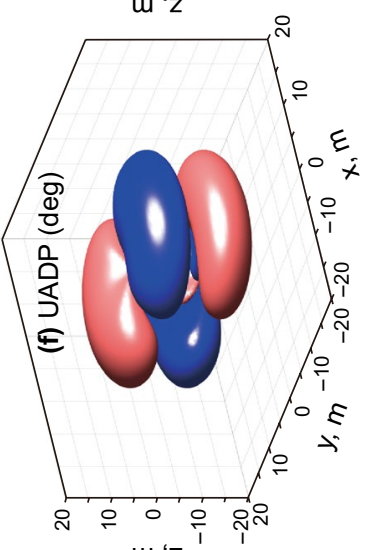

$m$ ' $z$

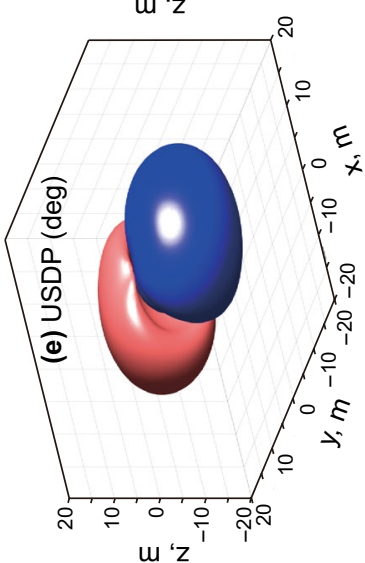

Fig. 2 Isosurfaces of EDARM geometrical factors

\subsection{Depth of detection (DoD)}

The boundary detection capability of EDARM mainly comes from Mode 1, and, therefore only USDA is considered in this subsection. To quantify the DoD of EDRAM, we consider a two-layered formation where the tool is parallel to the bed boundary. The noise-threshold-based definition of DoD has been widely applied in the industry. The maximum DoD is normally defined as the distance to the bed boundary when the amplitude of the response equals $0.25 \mathrm{~dB}$. By changing the resistivity besides the bed boundary, we can obtain the noise-threshold-based "Picasso" plot (Wang et al. 2018a). When the T-R spacing and the operating frequency are set to $13.72 \mathrm{~m}$ and $12 \mathrm{kHz}$, the USDA "Picasso" plot is shown in Fig. 3a. We arrive at the following conclusions: (1) the detection capability of USDA in a resistive bed is much larger than that obtained from a conductive layer; (2) the maximum DoD of USDA is $24.9 \mathrm{~m}$; (3) when the resistivity contrast is not big enough, there may exist a "blind zone" where the EDARM tools lose sensitivity to the bed boundary.

Although Fig. 3a has given the relationship between detection capability and formation resistivity, the noisethreshold-based DoD definition is unable to quantify the uncertainty of the tool's detection capability. Hence, an uncertainty-based definition of DoD is proposed in this subsection. Assuming the distance between the tool and the bed boundary is $D$, the simulated tool response vector is $\boldsymbol{M}$. If $+0.25 \mathrm{~dB}$ and $-0.25 \mathrm{~dB}$ noise are added to USDA data, the corresponding new data are $\boldsymbol{M}_{1}$ and $\boldsymbol{M}_{\mathbf{2}}$, respectively. Then, a Gauss-Newton iteration is performed once for the two new data sets. Here, the initial state is set to $D$. Then, the inverted distances $\left(D_{1}\right.$ and $\left.D_{2}\right)$ can be expressed as follows:

$D_{1}=D-\frac{\boldsymbol{J} \cdot\left(\boldsymbol{M}-\boldsymbol{M}_{1}\right)}{\boldsymbol{J}^{T} \boldsymbol{J}}, \quad D_{2}=D-\frac{\boldsymbol{J} \cdot\left(\boldsymbol{M}-\boldsymbol{M}_{2}\right)}{\boldsymbol{J}^{T} \boldsymbol{J}}$

where $\boldsymbol{J}$ is the Jacobian matrix and the superscript " $T$ " depicts the transpose operator of the matrix. The uncertainty factor $G$ of the inverted result is defined as

$G=\frac{\operatorname{abs}\left(D_{1}-D_{2}\right)}{D} \times 100 \%$

If the maximum DoD is defined as the distance to bed boundary when the uncertainty is $20 \%$, the uncertaintybased "Picasso" plot is shown in Fig. 3b. It is obvious the difference between two types of "Picasso" plots is at the upper-left corner and near the diagonal. Compared with Fig. 3a, the uncertainty-based "Picasso" plot has a smaller DoD and a relatively larger "blind zone." Moreover, the maximum DoD has reduced to $20.5 \mathrm{~m}$. 

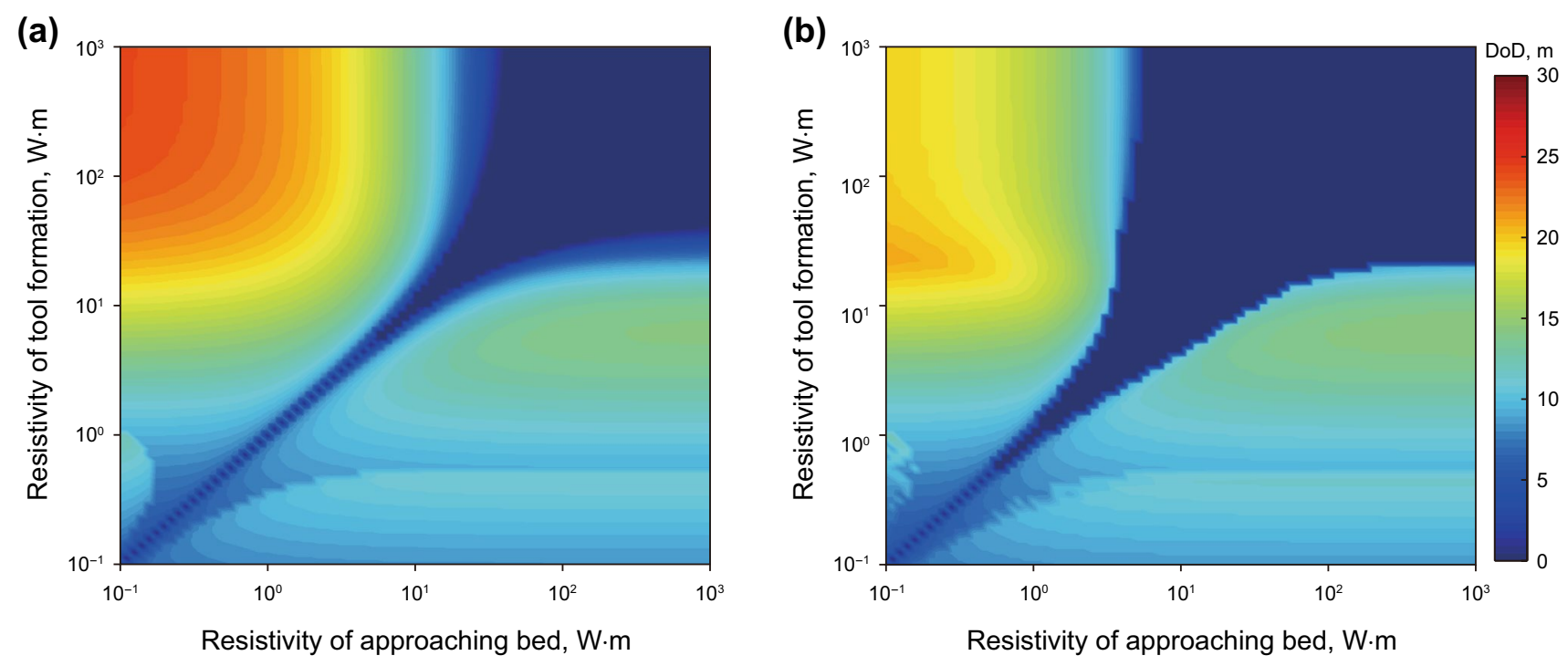

Fig. 3 a Noise-threshold-based "Picasso" plot; b uncertainty-based "Picasso" plot

By using the new DoD definition, Fig. 4 shows the relationship between detection capability versus spacing and operating frequency. We assume the tool is in sandstone with resistivity $100 \Omega \mathrm{m}$ and the surrounding bed is $0.1 \Omega \mathrm{m}$ shale. We can observe that the maximum DoD increases with increasing T-R spacing. By contrast, the maximum DoD may vary nonlinearly as the operating frequency becomes larger. When the T-R spacing is less than $30 \mathrm{~m}$, the maximum DoD can be up to $46.4 \mathrm{~m}$.

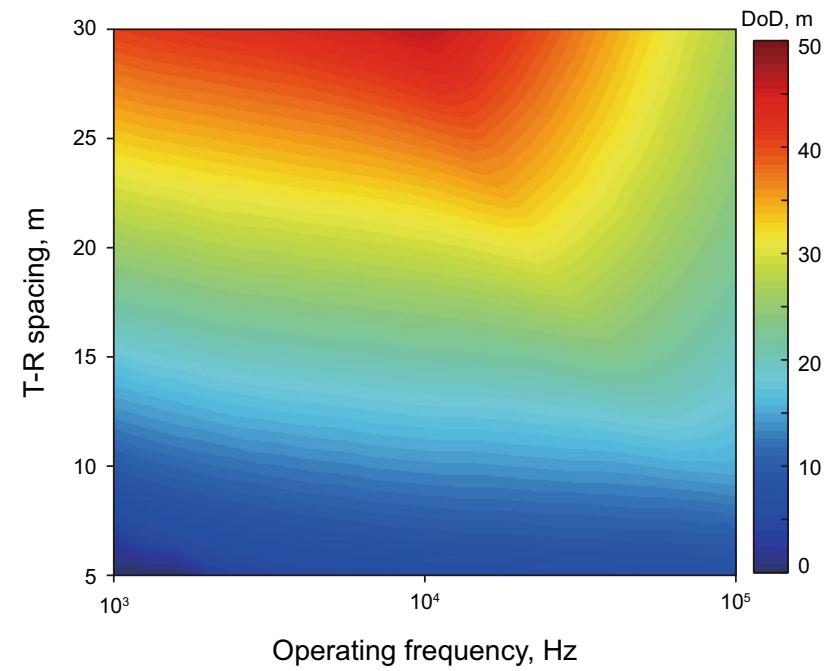

Fig. 4 The maximum DoD of USDA as a function of T-R spacing and operating frequency

\subsection{Resistivity range of use}

The resistivity range of use is another important factor to quantify the tool's detection performance (Li 2014). This parameter is normally affected by the T-R spacing and operating frequency. To obtain the resistivity range of use of EDARM, Fig. 5 shows the UHRA and UHRP responses as a function of formation resistivity in a homogeneous medium. In Fig. 5, three T-R spacings (i.e., $5 \mathrm{~m}, 18 \mathrm{~m}$ and $35 \mathrm{~m}$ ) are used and the corresponding operating frequencies are set to $2 / 6 \mathrm{kHz}, 12 / 24 \mathrm{kHz}$ and $48 / 96 \mathrm{kHz}$, respectively. As we can see, the UHRP is applicable to a formation with resistivity ranging from 0.1 to $1000 \Omega \mathrm{m}$. By contrast, UHRA is sensitive to the beds with resistivity less than $100 \Omega \mathrm{m}$ and its resistivity range of use enlarges with increasing operating frequency. It should be noted that the UHRP response may vary nonlinearly with the formation resistivity when the combination of long T-R spacing and relative high operating frequency is used.

\subsection{Sensitivity to resistivity anisotropy}

To investigate the sensitivity of EDARM to resistivity anisotropy, the EDARM response as a function of resistivity anisotropy $\lambda\left(\lambda=\sqrt{R_{h} / R_{v}}\right)$ and relative dip angle is presented in Fig. 6 where the horizontal resistivity is $1.0 \Omega \mathrm{m}$. Since the USDA and USDP are zero in a homogeneous formation, only the remaining modes are considered. From Fig. 6, we can see that there is a linear relationship between EDARM response and formation anisotropy. For Mode 3 and Mode 4, their amplitudes vary monotonously with the relative dip. By contrast, with the increase in relative dip, 

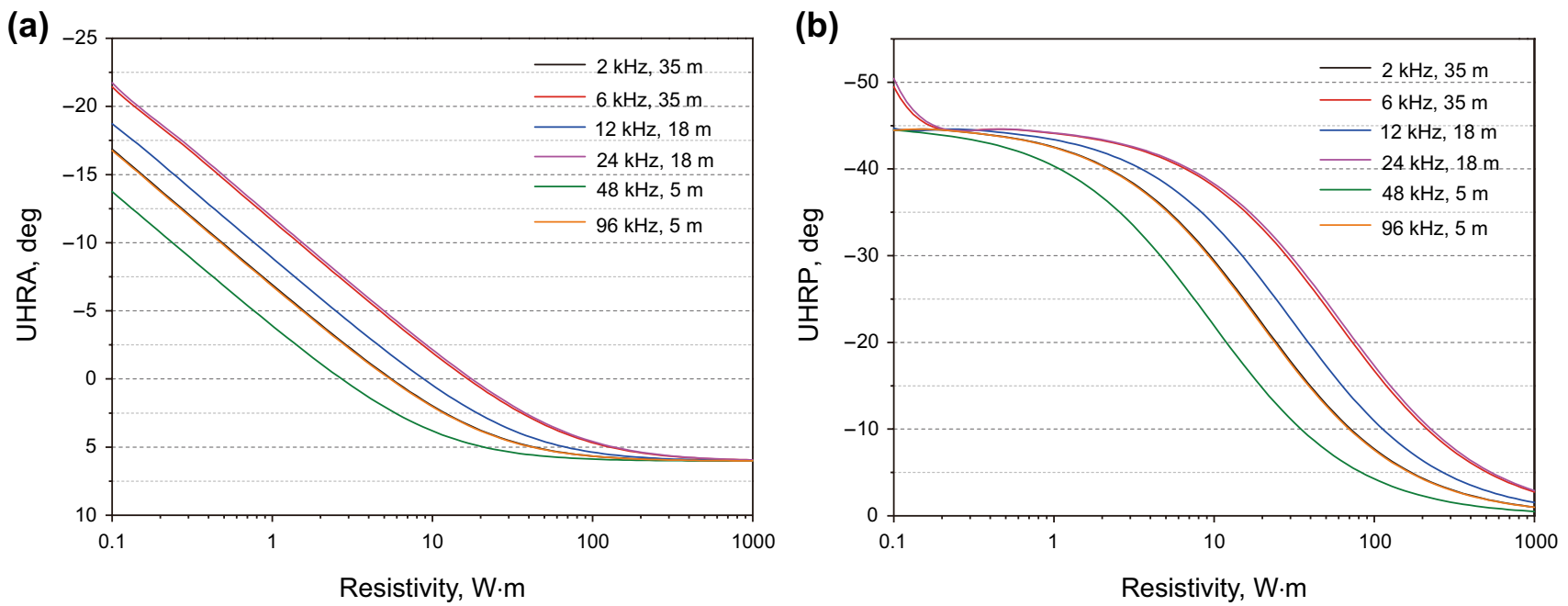

Fig. 5 Responses of UHRA and UHRP as a function of resistivity in a homogeneous formation

the amplitudes of UADA and UADP measurements increase first and decrease afterward.

Figure 7 shows the sensitivity of EDARM to formation anisotropy in arbitrary relative dips. Here, the sensitivity is defined as the differentiation of the EDARM response with respect to the anisotropy coefficient. Obviously, UADA and UADP measurements have the strongest sensitivity in a medium-deviated well $\left(30^{\circ}-70^{\circ}\right)$, whereas they almost lose sensitivity in near-vertical and horizontal wells. By contrast, UHAA and UHAP are sensitive to anisotropy in high-angle wells. By incorporating the $x x, y y$ and $z z$ components, the UHRA and UHRP measurements have guaranteed the sensitivity to anisotropy in a medium-deviated well. Overall, EDARM is capable of providing formation anisotropy in an arbitrary well deviation by jointly using the different measurements.

\section{Inversion and application of EDARM}

Despite the rich formation information conveyed, the interpretation of the EDARM data remains an open challenge, since the EDARM response is extremely complicated due to the effects of multiple bed boundaries. Therefore, inversion is required to quantitatively evaluate the formation structure (Bakr et al. 2017; Puzyrev et al. 2018). However, the large DoD of EDARM not only results in increasing parameters of interests, but also leads to abundant local minima in the cost function (Abubakar et al. 2008; Deng et al. 2012; Hong et al. 2013; Liu et al. 2015; Wang et al. 2018c; Wang and Fan 2019). What is worse, the lack of enough prior information further adds to the difficulty of the inversion and the traditional gradient optimization algorithm is no longer applicable (Habashy and Abubakar 2004). To address this problem, the stochastic Bayesian method associated with the MCMC sampling algorithm is introduced to extract the boundary positions and formation resistivity.

\subsection{Bayesian inversion theory}

In a Bayesian framework, the solution can be regarded as a set of random variables which satisfy a specific probability density function, namely posterior distribution (Sambridge 2014). Let the $\mathbf{d}^{\text {obs }}$ be the observed data and let $\mathbf{m}$ denote the model vector of inverted parameters, the posterior probability distribution $p\left(\mathbf{m} \mid \mathbf{d}^{\text {obs }}\right)$ can be formulated as

$p\left(\mathbf{m} \mid \mathbf{d}^{\mathrm{obs}}\right) \propto p\left(\mathbf{d}^{\mathrm{obs}} \mid \mathbf{m}\right) p(\mathbf{m})$

where $p(\mathbf{m})$ and $p\left(\mathbf{d}^{\text {obs }} \mid \mathbf{m}\right)$ depict the prior model distribution and the model likelihood, respectively. Assuming the prior distribution follows a uniform distribution and the likelihood satisfies Gaussian distribution, Eq. 8 can be written as

$p\left(\mathbf{m} \mid \mathbf{d}^{\mathrm{obs}}\right) \propto p\left(\mathbf{d}^{\mathrm{obs}} \mid \mathbf{m}\right) \propto \mathrm{e}^{-\frac{1}{2}\left[\mathbf{d}^{\mathrm{obs}}-\mathbf{S}(\mathbf{m})\right]^{T}\left[\mathbf{d}^{\mathrm{obs}}-\mathbf{S}(\mathbf{m})\right]}$

According to Eq. 9, the solution of the inverse problem is equal to reconstructing the likelihood function. To this end, the MCMC sampling algorithm is introduced. The kernel of MCMC is to construct a Markov Chain which satisfies the following detailed local balance

$p\left(\mathbf{x} \mid \mathbf{d}^{\text {obs }}\right) q(\mathbf{x}, \mathbf{y})=p\left(\mathbf{y} \mid \mathbf{d}^{\text {obs }}\right) q(\mathbf{y}, \mathbf{x})$

where $\mathbf{x}$ and $\mathbf{y}$ are arbitrary model vectors. $p\left(\mathbf{x} \mid \mathbf{d}^{\text {obs }}\right)$ is the marginal distribution of the Markov Chain and $q(\mathbf{x}, \mathbf{y})$ is a symmetric transition kernel function. By introducing the acceptance probability function $\alpha(\mathbf{y}, \mathbf{x})$ to Eq. 8 and using the "Metropolis-Hastings" algorithm (Metropolis et al. 1953), we have 
(a) UADA/dB

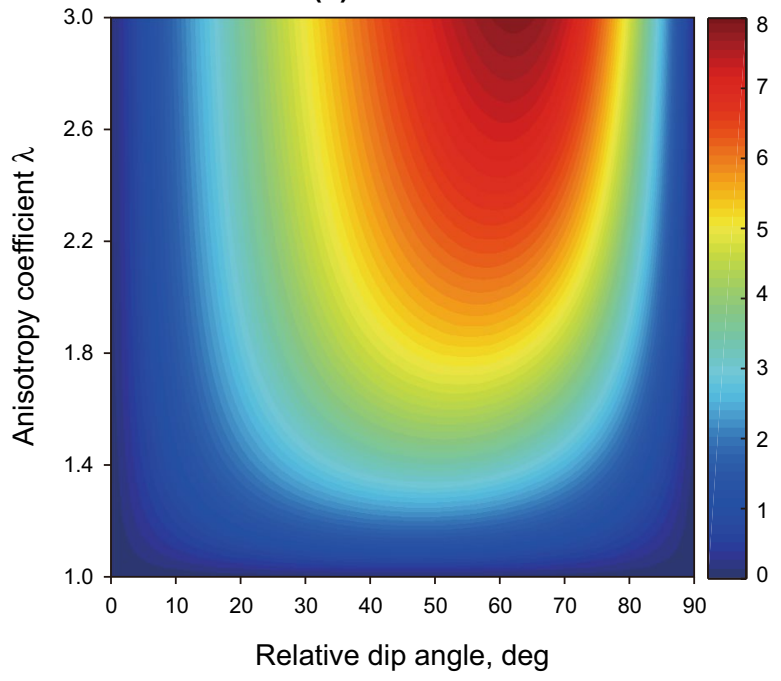

(c) UHAA/dB

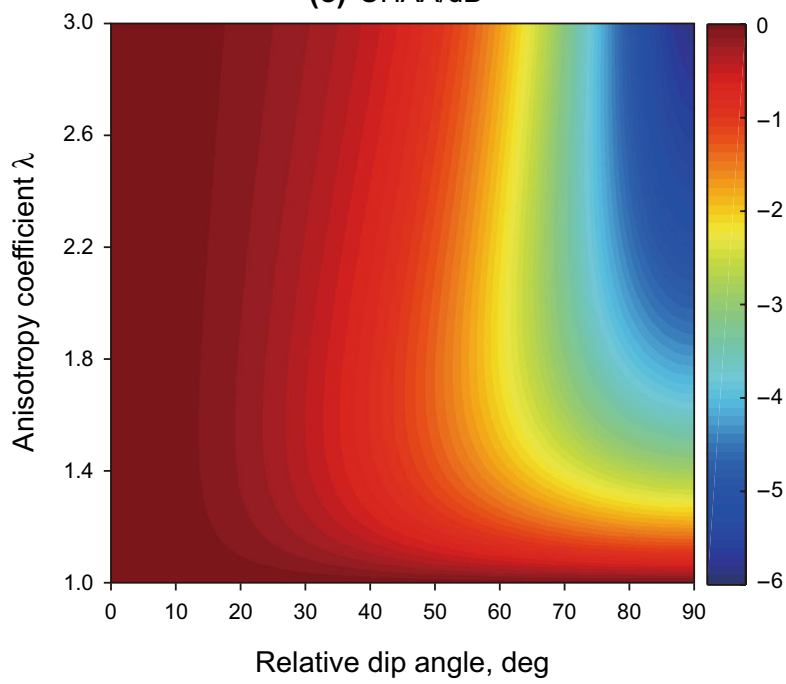

(e) UHRP/deg

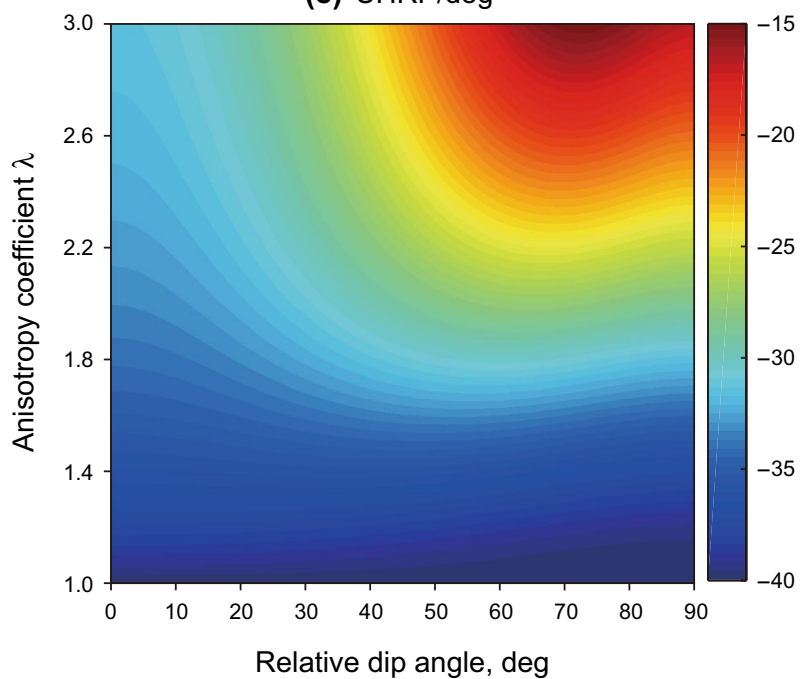

(b) UHRA/dB

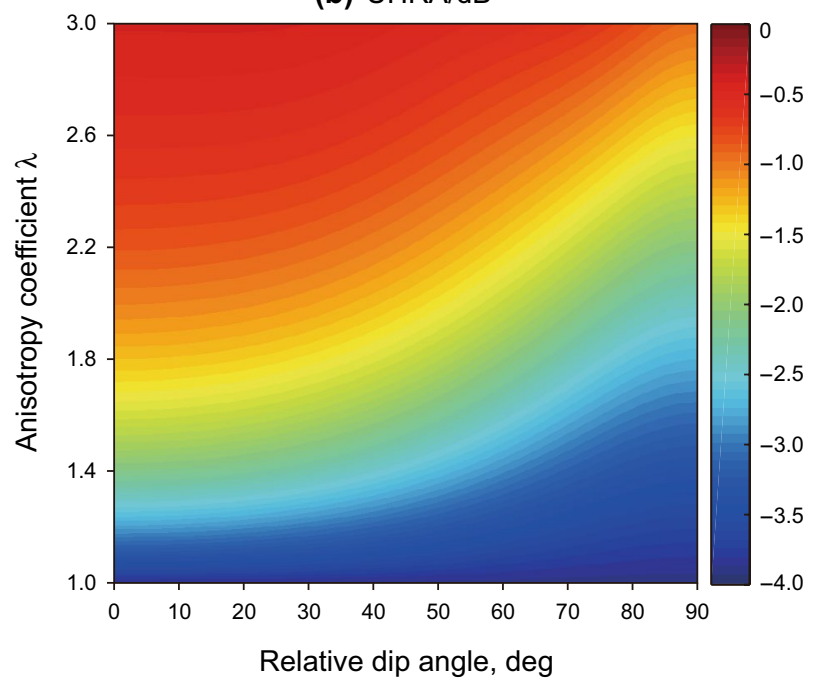

(d) UADP/deg

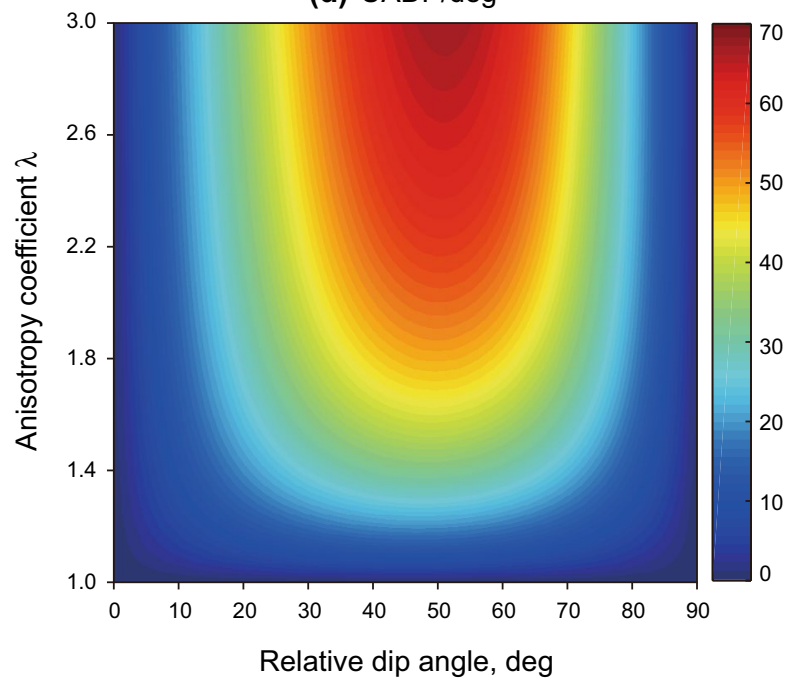

(f) UHAP/deg

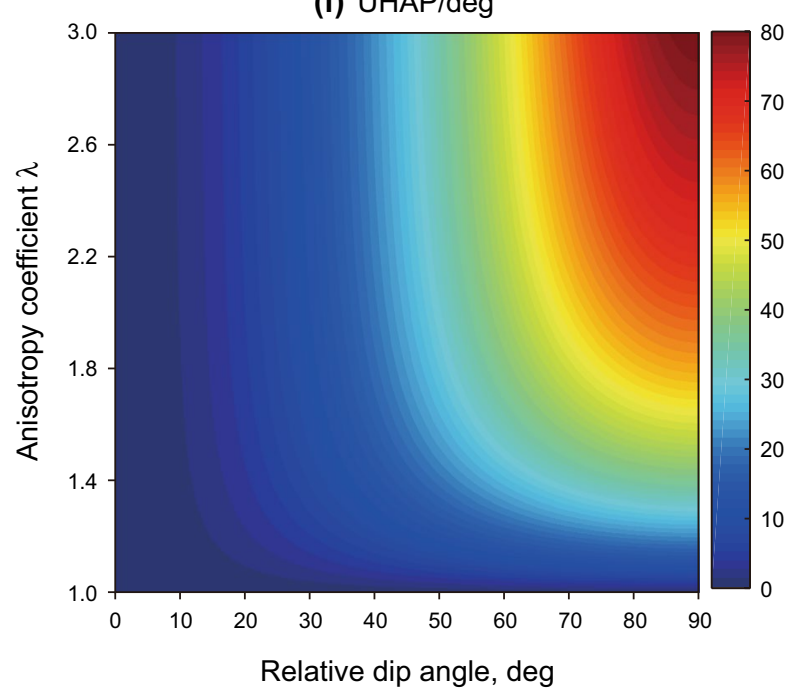

Fig. 6 EDARM response as a function of relative dip angle and anisotropy coefficient. The T-R spacing is $10 \mathrm{~m}$ and operating frequency is $12 \mathrm{kHz}$ 
(a) $\partial \mathrm{UADA} / \partial \lambda$

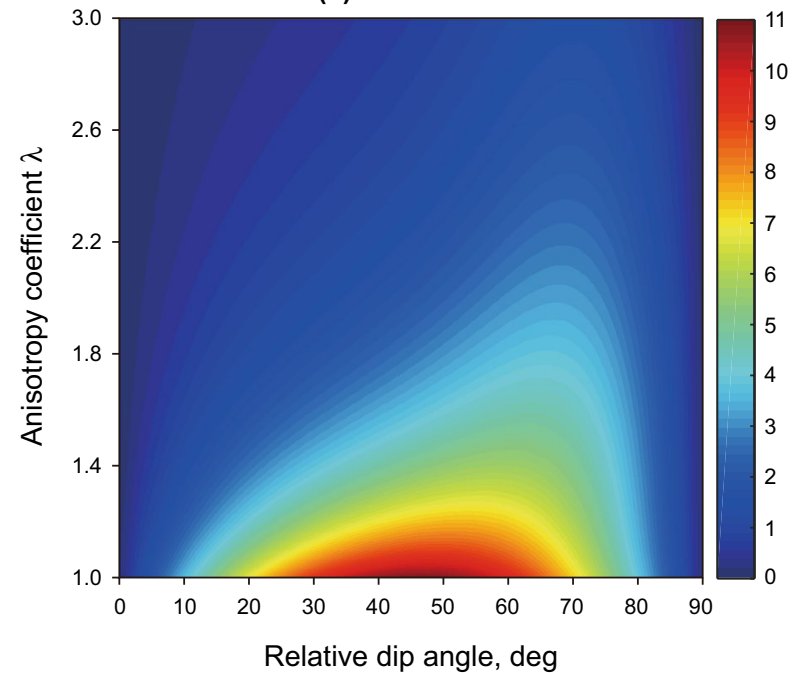

(c) $\partial \mathrm{UHAA} / \partial \lambda$

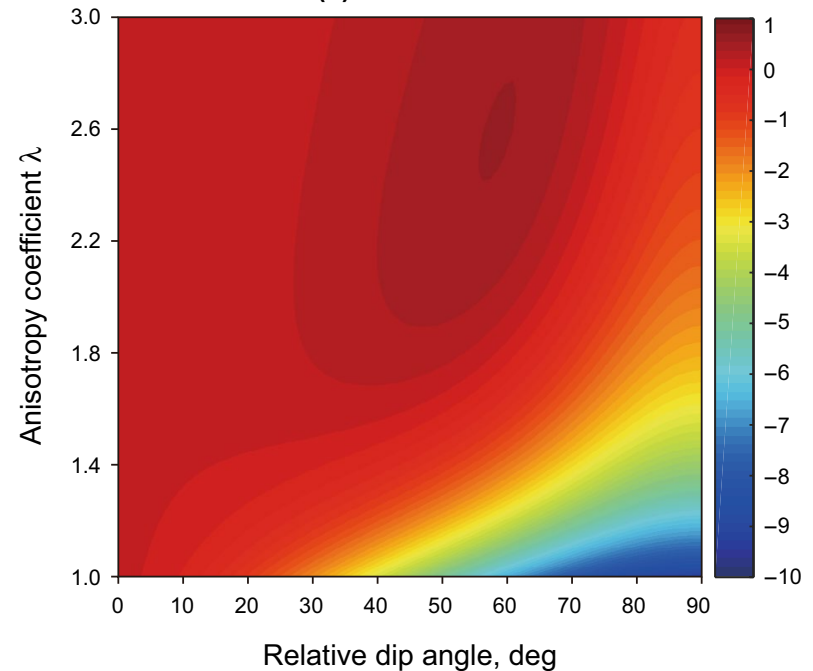

(e) $\partial \mathrm{UHRP} / \partial \lambda$

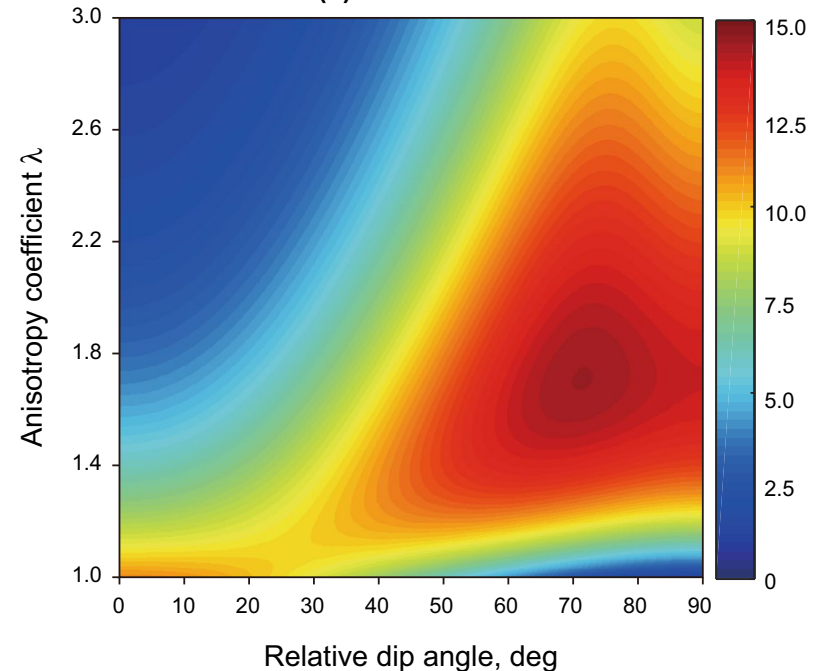

(b) $\partial \mathrm{UHRA} / \partial \lambda$

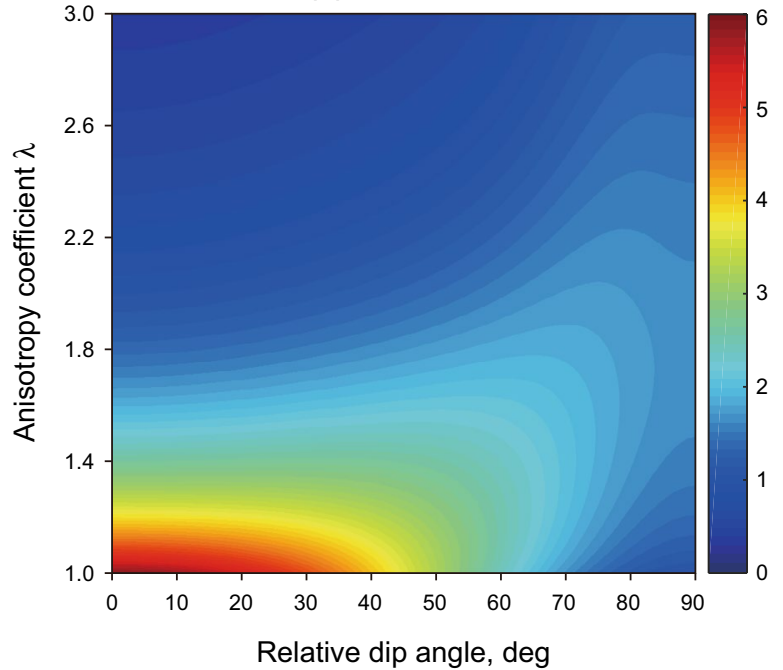

(d) $\partial U A D P / \partial \lambda$

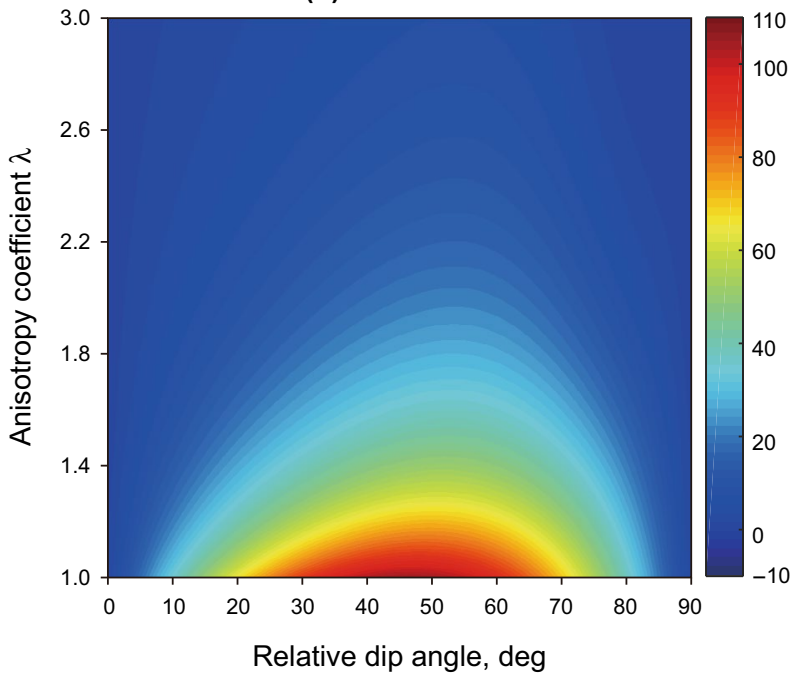

(f) $\partial \mathrm{UHAP} / \partial \lambda$

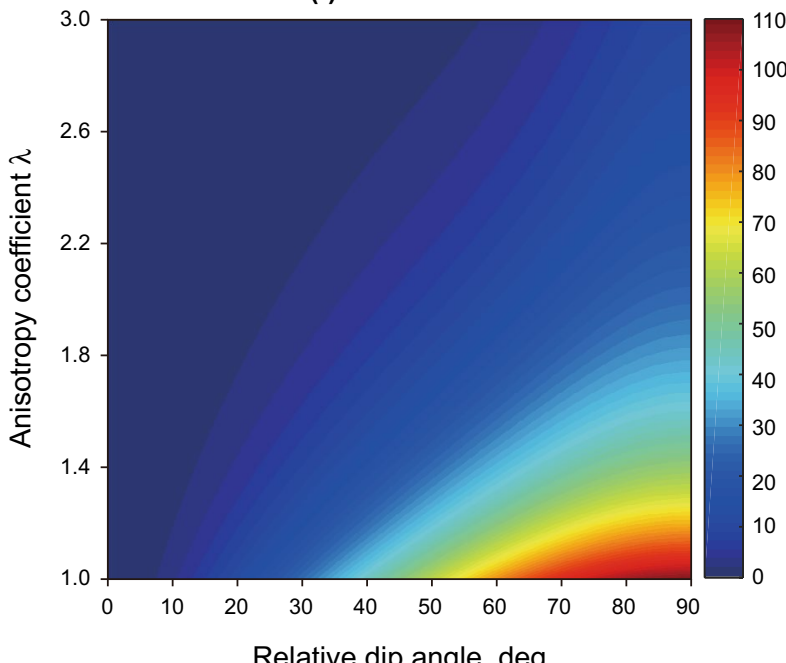

Relative dip angle, deg

Fig. 7 Sensitivity of EDARM to resistivity anisotropy 
$p\left(\mathbf{x} \mid \mathbf{d}^{\mathrm{obs}}\right) q(\mathbf{x}, \mathbf{y}) \alpha(\mathbf{x}, \mathbf{y})=p\left(\mathbf{y} \mid \mathbf{d}^{\mathrm{obs}}\right) q(\mathbf{y}, \mathbf{x}) \alpha(\mathbf{y}, \mathbf{x})$

where

$\alpha(\mathbf{x}, \mathbf{y})=\min \left\{1, \frac{p\left(\mathbf{d}^{\mathrm{obs}} \mid \mathbf{y}\right)}{p\left(\mathbf{d}^{\mathrm{obs}} \mid \mathbf{x}\right)}\right\}, \quad \alpha(\mathbf{y}, \mathbf{x})=\min \left\{1, \frac{p\left(\mathbf{d}^{\mathrm{obs}} \mid \mathbf{x}\right)}{p\left(\mathbf{d}^{\mathrm{obs}} \mid \mathbf{y}\right)}\right\}$

\subsection{Synthetic example 1: geosteering application}

In this subsection, the benchmark model A5 developed by the Standardization of LWD Deep Azimuthal Resistivity work group (SDAR) is used to verify the feasibility and accuracy of the stochastic inversion method. As shown in Fig. $8 \mathrm{a}$, the benchmark model consists of a $2.0 \Omega \mathrm{m}$ shale bed and a sandstone with resistivity $100 \Omega \mathrm{m}$. The tool drills across the beds from top to bottom with a fixed entry angle of $78^{\circ}$. Figure $8 \mathrm{~b}, \mathrm{c}$ shows the measured EDARM responses when the T-R spacing is $15.0 \mathrm{~m}$ and the operating frequency is $12 \mathrm{kHz}$.

The aforementioned stochastic inversion method is applied to derive the true formation parameters, and $3 \times 10^{5}$ samples are drawn randomly in each inversion. The inverted two-dimensional (2D) resistivity curtain from the EDARM data with $15.0 \mathrm{~m}$ spacing and $12 \mathrm{kHz}$ operating frequency is shown in Fig. 9a where the color of each pixel depicts the formation resistivity. Compared with Fig. 8a, the inverted 2D curtain agrees well with the true formation model, indicating the accuracy and robustness of the inversion. The smaller the distance between the tool and bed boundary is, the more reliable the estimated boundary and formation resistivity are.
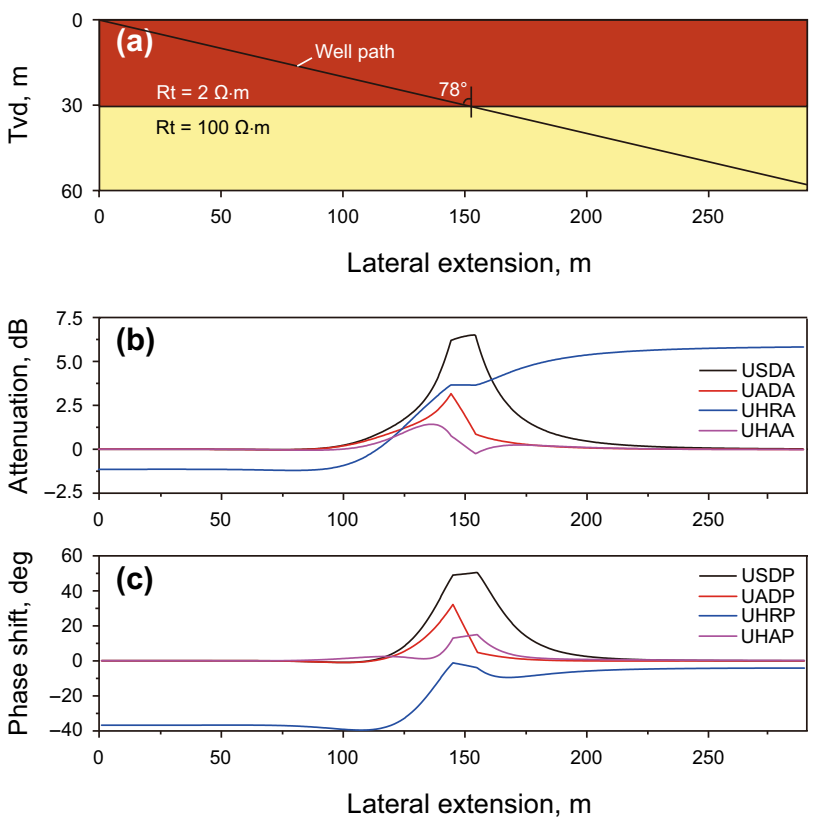

Fig. 8 a The SDAR benchmark model A5; b measured attenuation EDARM responses; $\mathbf{c}$ measured phase-shift EDARM responses

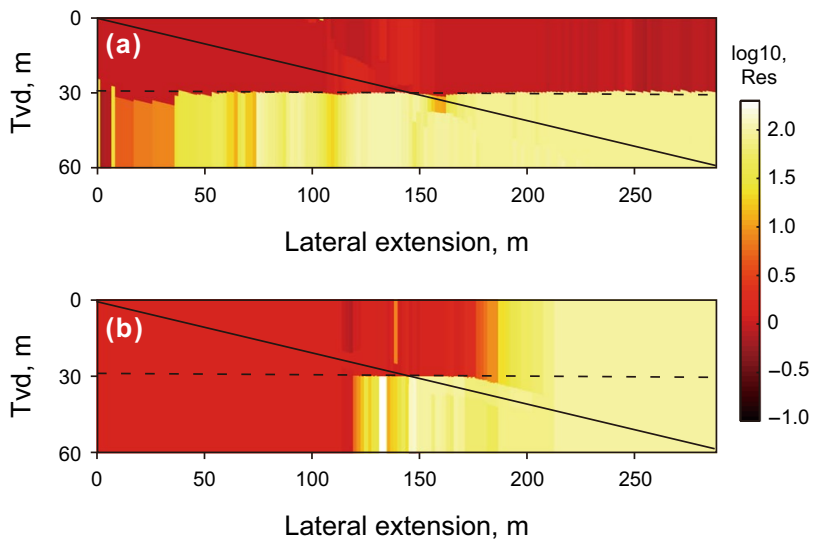

Fig. 9 a Inverted two-dimensional (2D) resistivity curtain from LWD EDARM data; $\mathbf{b}$ inverted 2D resistivity curtain from LWD ARM data

When the tool is in a resistive layer, a bed boundary which is less than $25 \mathrm{~m}$ around the borehole can be readily obtained. On the contrary, the reliable inverted distance to boundary reduces to $17 \mathrm{~m}$ when the tool lies in a conductive formation. This is because the EDARM responses are very sensitive to the nearby conductive surrounding bed and the tool has better detection capability in a resistive bed. It is worth mentioning that the resistivity curtain can still be obtained even though the remote boundary is beyond the tool's maximum DoD. In this specific case, the extracted boundary is not reliable and can even be inaccurate.

To further demonstrate the landing and geosteering capability of EDARM, Fig. 9b presents the inverted 2D resistivity curtain derived from LWD ARM data. Obviously, ARM tool is capable of providing a bed boundary up to $5 \mathrm{~m}$ around the borehole. When the ARM tool is in the conductive bed, there may be strong uncertainty in the estimated surrounding bed resistivity. By contrast, when the tool lies within a resistive formation, the precision of the estimated boundary reduces as the distance to bed boundary increases. From Fig. 9, we can conclude that the detection capability of EDARM to a remote bed boundary is several times deeper, which has significantly improved the geosteering application.

\subsection{Synthetic example 2: reservoir imaging}

In this subsection, a folded model shown in Fig. 10a is established to showcase the capability of EDARM for multiple boundary detection and formation structure imaging. The model consists of 10 anisotropic beds and the formation parameters are specified in Table 1. When the tool penetrates the beds from top to bottom, the observed EDARM attenuation curves with T-R $5.0 \mathrm{~m}$ spacing and $48 \mathrm{kHz}$ operating frequency are displayed in Fig. 10b. The corresponding inverted result is shown in Fig. 11a. It is clear that multiple bed boundaries can be readily obtained and the precision 

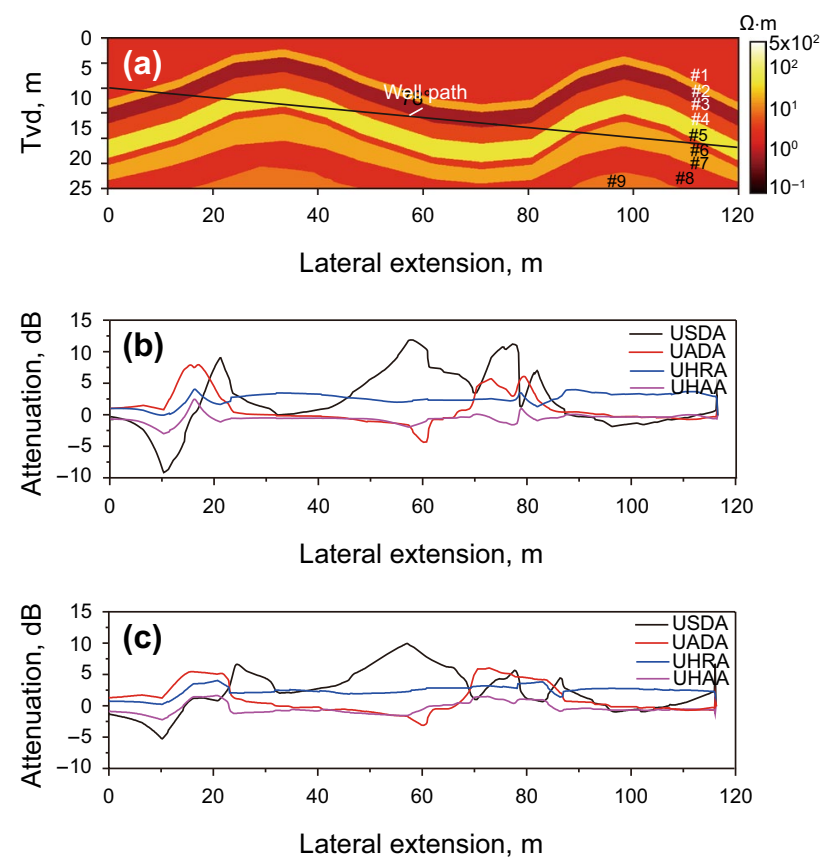

Fig. 10 a The folded formation model; $\mathbf{b}$ the measured attenuation EDARM curves with T-R spacing $5.0 \mathrm{~m}$ and operating frequency $48 \mathrm{kHz}$; $\mathbf{c}$ the measured attenuation EDARM curves with T-R spacing $10.0 \mathrm{~m}$ and operating frequency $12 \mathrm{kHz}$

of inverted boundary positions is improved when the tool approaches the boundary. Moreover, in the section with lateral extension 0-60 m, the formation structure can be readily derived. However, the low-resistivity layer (layer 2) above the well path at lateral extension $80-120 \mathrm{~m}$ is unavailable. This can be explained as the remote bed boundaries are far beyond the tool's detection capability.

When the T-R spacing is $10 \mathrm{~m}$ and operating frequency is set to $12 \mathrm{kHz}$, the EDARM attenuation response and corresponding inversion result are shown in Figs. 10c and 11b, respectively. From Fig. 11b, we can observe that the overlying conductive bed (layer \#2) at lateral extension $100 \mathrm{~m}$ can still be obtained when the T-R spacing is $10 \mathrm{~m}$, which shows the improved detection capability. In addition, the whole distribution of the formation structure is readily recognized. Compared with Fig. 11a, the main disadvantage of
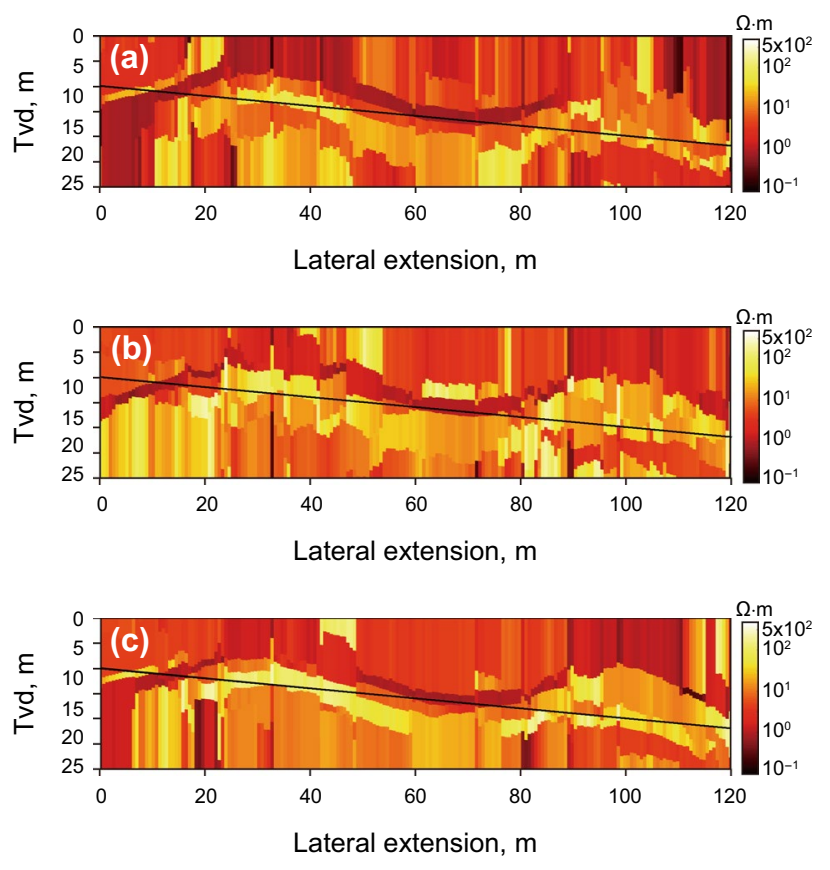

Fig. 11 The inverted 2D resistivity curtain for the EDARM data with a $5.0 \mathrm{~m}$ T-R spacing and $48 \mathrm{kHz}$ operating frequency; b $10.0 \mathrm{~m}$ T-R spacing and $12 \mathrm{kHz}$ operating frequency; c $5.0 \mathrm{~m}$ T-R spacing, $48 \mathrm{kHz}$ operating frequency and $10.0 \mathrm{~m}$ T-R spacing, $12 \mathrm{kHz}$ operating frequency

inverted result from the long-spacing EDARM data is the low imaging resolution. If the data in Fig. 10b, c are used together for a joint inversion, the inverted results are shown in Fig. 11c. Compared with the result from single-spacing data, the joint inversion not only guarantees the high resolution of the resistivity curtain, but also enables the deep detection of the remote bed boundaries.

\section{Conclusions}

1. USDA and USDP show the strongest sensitivity to bed boundaries, whereas UADA and UADP are specifically designed for the detection of relative dips and the resistivity anisotropy coefficient. The geometrical factors of
Table 1 Specifications of each bed of the folding model

\begin{tabular}{lcclcc}
\hline $\begin{array}{l}\text { Layer } \\
\text { number }\end{array}$ & $\begin{array}{l}\text { Horizontal resistiv- } \\
\text { ity, } \Omega \mathrm{m}\end{array}$ & $\begin{array}{l}\text { Vertical resistiv- } \\
\text { ity, } \Omega \mathrm{m}\end{array}$ & $\begin{array}{l}\text { Layer } \\
\text { number }\end{array}$ & $\begin{array}{l}\text { Horizontal resistiv- } \\
\text { ity, } \Omega \mathrm{m}\end{array}$ & $\begin{array}{l}\text { Vertical resistiv- } \\
\text { ity, } \Omega \mathrm{m}\end{array}$ \\
\hline$\# 1$ & 3.0 & 6.0 & $\# 6$ & 4.0 & 8.0 \\
$\# 2$ & 20.0 & 40.0 & $\# 7$ & 20.0 & 50.0 \\
$\# 3$ & 0.8 & 2.0 & $\# 8$ & 3.0 & 5.0 \\
$\# 4$ & 5.0 & 10.0 & $\# 9$ & 10.0 & 10.0 \\
$\# 5$ & 50.0 & 50.0 & & & \\
\hline
\end{tabular}


UHRA and UHRP are axially symmetric with respect to the $\mathrm{z}$-axis and thus provide non-azimuthal formation resistivity. By contrast, UHAA and UHAP are able to detect the anisotropy coefficient by using the difference between the two coplanar components.

2. The new proposed uncertainty-based DoD definition, which considers the effect of data noise on the inversion results, can provide more reliable and practical assessment of the tool's boundary detection capability. The maximum reliability of EDARM increases with the increasing T-R spacing, while it may vary nonlinearly as the operating frequency becomes larger.

3. UHRP can accommodate a formation with resistivity ranging from $0.1 \Omega \mathrm{m}$ to $1000 \Omega \mathrm{m}$, while UHRA is only sensitive to the beds with resistivity less than $100 \Omega \mathrm{m}$. By jointly using the different measurements, EDARM is capable of providing formation anisotropy in arbitrary well deviations.

4. Compared with ARM tool, the detection capability of EDARM is several times deeper, which has significantly improved the geosteering application. Moreover, taking advantage of the Bayesian inversion method and EDARM data, the multiple boundaries and the resistivity distributions of the reservoir structure around the borehole can be readily detected.

Acknowledgements This research has been co-funded by Chinese Postdoctoral Science Foundation (2018M640663), the National Natural Science Foundation of China $(41474100,41574118,41674131)$ and National Science and Technology Major Project of the Ministry of Science and Technology of China (2017ZX05009-001).

Open Access This article is distributed under the terms of the Creative Commons Attribution 4.0 International License (http://creativeco mmons.org/licenses/by/4.0/), which permits unrestricted use, distribution, and reproduction in any medium, provided you give appropriate measured voltage $V\left(\mathrm{r}_{R} ; \mathrm{r}_{T}\right)$ at the receiver can be regarded as the summation of the incident field $V_{b}$ irradiated in background medium and the scattered field caused by the inhomogeneous geometry

$V\left(\mathbf{r}_{R} ; \mathbf{r}_{T}\right)=V_{b}\left(\mathbf{r}_{R} ; \mathbf{r}_{T}\right)+\int_{V} d \mathbf{r}^{\prime} \delta \sigma\left(\mathbf{r}^{\prime}\right) \boldsymbol{G}\left(\mathbf{r}_{R} ; \mathbf{r}^{\prime}\right) V\left(\mathbf{r}^{\prime}\right)$

where $\boldsymbol{G}$ is the Green's function and $\delta \sigma\left(\mathbf{r}^{\prime}\right)$ depicts the conductivity difference between background medium and the inhomogeneous geometry, $\delta \sigma\left(\mathbf{r}^{\prime}\right)=\sigma\left(\mathbf{r}^{\prime}\right)-\sigma_{b}$. Using the Born approximation, the electrical field at the abnormality is linearly approximated and thus Eq. 13 can be written as

$V\left(\mathbf{r}_{R} ; \mathbf{r}_{T}\right)=V_{b}\left(\mathbf{r}_{R} ; \mathbf{r}_{T}\right)+\int_{V} d \mathbf{r}^{\prime} \delta \sigma\left(\mathbf{r}^{\prime}\right) \boldsymbol{G}\left(\mathbf{r}_{R} ; \mathbf{r}^{\prime}\right) V_{b}\left(\mathbf{r}^{\prime}\right)$

To simplify the formula derivation, we assume each mode of EDARM can be equivalent to two voltages $\left(V_{1}\right.$ and $\left.V_{2}\right)$. Thus the phase shift $\Delta \phi$ and attenuation $S$ between the two voltages are defined as

$\frac{V_{1}\left(\mathbf{r}_{R} ; \mathbf{r}_{T}\right)}{V_{2}\left(\mathbf{r}_{R} ; \mathbf{r}_{T}\right)}=S \cdot e^{i \Delta \phi} \Leftrightarrow \ln V_{1}-\ln V_{2}=\ln S+i \Delta \phi$

Differentiating the above equation on both sides yields

$\frac{\delta V_{1}}{V_{1}}-\frac{\delta V_{2}}{V_{2}}=\delta \ln S+i \delta \Delta \phi$

After simplification, we have

$\delta \ln S=\operatorname{Re}\left(\frac{\delta V_{1}}{V_{1}}-\frac{\delta V_{2}}{V_{2}}\right) \quad \delta \Delta \phi=\operatorname{Im}\left(\frac{\delta V_{1}}{V_{1}}-\frac{\delta V_{2}}{V_{2}}\right)$

where $\operatorname{Re}(\cdot)$ and $\operatorname{Im}(\cdot)$ are the functions to derive the real and imaginary part of a complex value, respectively. Substituting Eq. (18) into Eq. (14) yields

$\delta \ln S=\operatorname{Re}\left(\frac{\int_{V} d \mathbf{r}^{\prime} \delta \sigma\left(\mathbf{r}^{\prime}\right) \boldsymbol{G}\left(\mathbf{r}_{R 1} ; \mathbf{r}^{\prime}\right) V_{b}\left(\mathbf{r}^{\prime}\right)}{V_{b 1}}-\frac{\int_{V} d \mathbf{r}^{\prime} \delta \sigma\left(\mathrm{r}^{\prime}\right) \boldsymbol{G}\left(\mathbf{r}_{R 2} ; \mathbf{r}^{\prime}\right) V_{b}\left(\mathbf{r}^{\prime}\right)}{V_{b 2}}\right)$
$\delta \Delta \phi=\operatorname{Im}\left(\frac{\int_{V} d \mathbf{r}^{\prime} \delta \sigma\left(\mathbf{r}^{\prime}\right) \boldsymbol{G}\left(\mathbf{r}_{R 1} ; \mathbf{r}^{\prime}\right) V_{b}\left(\mathbf{r}^{\prime}\right)}{V_{b 1}}-\frac{\int_{V} d \mathbf{r}^{\prime} \delta \sigma\left(\mathbf{r}^{\prime}\right) \boldsymbol{G}\left(\mathbf{r}_{R 2} ; \mathbf{r}^{\prime}\right) V_{b}\left(\mathbf{r}^{\prime}\right)}{V_{b 2}}\right)$

credit to the original author(s) and the source, provide a link to the Creative Commons license, and indicate if changes were made.

\section{Appendix}

Assuming the transmitter and receiver are at $\mathbf{r}_{T}\left(\rho_{T}, \phi_{T}, \mathbf{z}_{T}\right)$ and $\mathbf{r}_{R}\left(\rho_{R}, \phi_{R}, \mathbf{z}_{R}\right)$, the distance between transmitter and receiver is $L$. According to integral equation theory, the
Here, $\delta \ln S$ and $\delta \Delta \phi$ are the relative contributions of formation units to the attenuation and phase-shift measurements. Replacing the $V_{1}$ and $V_{2}$ of Eq. 17 with the detailed expression of EDARM modes, the corresponding geometrical factor can be derived. Taking Mode 1 for example, $V_{1}=\left(V_{z z}-V_{z x}\right)\left(V_{z z}+V_{x z}\right), \quad V_{2}=\left(V_{z z}+V_{z x}\right)\left(V_{z z}-V_{x z}\right)$. The geometrical factor $\mathrm{g}_{\mathrm{USDA}}$ and $g_{\mathrm{USDP}}$ are shown as follows 
$\mathrm{g}_{\mathrm{USDA}}=\operatorname{real}\left\{\frac{\delta\left[\left(V_{z z}-V_{z x}\right)\left(V_{z z}+V_{x z}\right)\right]}{\left(V_{b z z}-V_{b z x}\right)\left(V_{b z z}+V_{b x z}\right)}-\frac{\delta\left[\left(V_{z z}+V_{z x}\right)\left(V_{z z}-V_{x z}\right)\right]}{\left(V_{b z z}+V_{b z x}\right)\left(V_{b z z}-V_{b x z}\right)}\right\}$
$g_{\mathrm{USDP}}=\operatorname{imag}\left\{\frac{\delta\left[\left(V_{z z}-V_{z x}\right)\left(V_{z z}+V_{x z}\right)\right]}{\left(V_{b z z}-V_{b z x}\right)\left(V_{b z z}+V_{b x z}\right)}-\frac{\delta\left[\left(V_{z z}+V_{z x}\right)\left(V_{z z}-V_{x z}\right)\right]}{\left(V_{b z z}+V_{b z x}\right)\left(V_{b z z}-V_{b x z}\right)}\right\}$

Equation 19 can be further simplified as

$g_{\mathrm{USDA}}=\operatorname{real}\left\{\frac{\frac{L^{2} \rho^{5}}{2 r_{R}^{6} r_{T}^{6}}[\cos \varphi(z+L / 2)-\sin \varphi(z-L / 2)]}{\left[\frac{1}{4 \pi L^{3}} e^{i k L}(-2 i k L+2)\right]^{2}}\left[\left(1-i k r_{R}\right)\left(1-i k r_{T}\right) e^{i k\left(r_{R}+r_{T}\right)}\right]^{2}\right\}$

$g_{\mathrm{USDP}}=\operatorname{imag}\left\{\frac{\frac{L^{2} \rho^{5}}{2 r_{R}^{6} r_{T}^{6}}[\cos \varphi(z+L / 2)-\sin \varphi(z-L / 2)]}{\left[\frac{1}{4 \pi L^{3}} e^{i k L}(-2 i k L+2)\right]^{2}}\left[\left(1-i k r_{R}\right)\left(1-i k r_{T}\right) e^{i k\left(r_{R}+r_{T}\right)}\right]^{2}\right\}$

Similarly, the analytical GF expressions of other EDARM

modes are readily obtained

$$
\begin{aligned}
& g_{\mathrm{UADA}}=\operatorname{real}\left\{\frac{\frac{L^{2} \rho^{5}}{2 r_{R}^{6} r_{T}^{6}}[\cos \varphi(z+L / 2)+\sin \varphi(z-L / 2)]}{\left[\frac{1}{4 \pi L^{3}} e^{i k L}(-2 i k L+2)\right]^{2}}\left[\left(1-i k r_{R}\right)\left(1-i k r_{T}\right) e^{i k\left(r_{R}+r_{T}\right)}\right]^{2}\right\} \\
& g_{\mathrm{UADP}}=\operatorname{imag}\left\{\frac{\frac{L^{2} \rho^{5}}{2 r_{R}^{6} r_{T}^{6}}[\cos \varphi(z+L / 2)+\sin \varphi(z-L / 2)]}{\left[\frac{1}{4 \pi L^{3}} e^{i k L}(-2 i k L+2)\right]^{2}}\left[\left(1-i k r_{R}\right)\left(1-i k r_{T}\right) e^{\left.\left.i k\left(r_{R}+r_{T}\right)\right]^{2}\right\}}\right\}\right. \\
& g_{\mathrm{UHRA}}=\operatorname{real}\left\{\frac{\pi L^{4} \rho}{r_{R}^{3} r_{T}^{3} e^{i k L}}\left[\frac{\rho^{2}}{-i k L+1}-\frac{\rho^{2}+2(z+L / 2)(z-L / 2)}{k^{2} L^{2}+i k L-1}\right]\left(1-i k r_{R}\right)\left(1-i k_{b} r_{T}\right) e^{i k\left(r_{R}+r_{T}\right)}\right\} \\
& g_{\mathrm{UHRP}}=\operatorname{imag}\left\{\frac{\pi L^{4} \rho}{r_{R}^{3} r_{T}^{3} e^{i k L}}\left[\frac{\rho^{2}}{-i k L+1}-\frac{\rho^{2}+2(z+L / 2)(z-L / 2)}{k^{2} L^{2}+i k L-1}\right]\left(1-i k r_{R}\right)\left(1-i k_{b} r_{T}\right) e^{i k\left(r_{R}+r_{T}\right)}\right\} \\
& g_{\mathrm{UHAA}}=\operatorname{real}\left\{\frac{2 \pi L^{4} \rho^{3}\left(\sin ^{2} \varphi-\cos ^{2} \varphi\right)}{r_{R}^{3} r_{T}^{3} e^{i k L}\left(k^{2} L^{2}+i k L-1\right)}\left(1-i k r_{R}\right)\left(1-i k_{b} r_{T}\right) e^{i k\left(r_{R}+r_{T}\right)}\right\} \\
& g_{\mathrm{UHAP}}=\operatorname{imag}\left\{\frac{2 \pi L^{4} \rho^{3}\left(\sin ^{2} \varphi-\cos ^{2} \varphi\right)}{r_{R}^{3} r_{T}^{3} e^{i k L}\left(k^{2} L^{2}+i k L-1\right)}\left(1-i k r_{R}\right)\left(1-i k_{b} r_{T}\right) e^{i k\left(r_{R}+r_{T}\right)}\right\}
\end{aligned}
$$

\section{References}

Abubakar A, Habashy TM, Druskin VL, Knizhnerman L, Alumbaugh D. 2.5D forward and inverse modeling for interpreting low-frequency electromagnetic measurements. Geophysics. 2008;73(4):F165-77. https://doi.org/10.1190/1.2937466.
Bakr SA, Pardo D, Torres-Verdin C. Fast inversion of logging-whiledrilling resistivity measurements acquired in multiple wells. Geophysics. 2017;82(3):E111-20. https://doi.org/10.1190/geo20 16-0292.1. 
Bittar M, Aki A. Advancement and economic benefit of geosteering and well-placement technology. Lead Edge. 2015;34(5):524-8. https://doi.org/10.1190/tle34050524.1.

Bittar MS, Klein JD, Randy B, Hu G, Wu M, Pitcher JL, et al. A new azimuthal deep-reading resistivity tool for geosteering and advanced formation evaluation. SPE Reservoir Eval Eng. 2009;12(2):270-9. https://doi.org/10.2118/109971-PA.

Chaumont-Frelet T, Pardo D, Rodriguez-Rozas A. Finite element simulations of logging-while-drilling and extra-deep azimuthal resistivity measurements using non-fitting grids. Comput Geosci. 2018;22(5):1161-74. https://doi.org/10.1007/s10596-018-9744-6.

Dupuis C, Denichou JM. Automatic inversion of deep-directionalresistivity measurements for well placement and reservoir description. Lead Edge. 2015;34(5):504-12. https://doi.org/10.1190/tle34 050504.1.

Deng SG, Sun QT, Li H, Huo N, He X. The sensitivity of the array resistivity $\log$ to mud filtrate invasion and its primary fiveparameter inversion for improved oil water recognition. Pet Sci. 2012;9(3):295-302. https://doi.org/10.1007/s12182-012-0212-y.

Gao J, Xu C, Xiao J. Forward modelling of multi-component induction logging tools in layered anisotropic dipping formations. J Geophys Eng. 2013;10:054007. https://doi.org/10.1088/17422132/10/5/054007.

Habashy TM, Abubakar A. A general framework for constraint minimization for the inversion of electromagnetic measurements. Prog Electromagn Res. 2004;46:265-312. https://doi.org/10.2528/ PIER03100702.

Hagiwara T. EM log response to anisotropic resistivity in thinly laminated formations with emphasis on 2-MHz resistivity devices. SPE Form Eval. 1996;11(4):211-7. https://doi.org/10.2118/28426 -PA.

Hong D, Yang S. Multi-component induction logging response in large dielectric formations. Acta Phys Sin. 2011;60(10):109101. https ://doi.org/10.1002/pssc.201000025 (in Chinese).

Hong D, Xiao JQ, Zhang G, Zhao Y, Liang X. Fast inverse the relative dip using cross-component in highly deviated well. Chin J Geophys. 2013;56(7):2494-501. https://doi.org/10.6038/cjg2013073 5 (in Chinese).

Li H, Wang H. Investigation of eccentricity effects and depth of investigation of azimuthal resistivity LWD tools using 3D finite difference method. J Petrol Sci Eng. 2016;143:211-25. https://doi. org/10.1016/j.petrol.2016.02.032.

Li JH. Integral equation of relationship on the apparent conductivity and true conductivity in induction logging. Sci China Earth Sci. 2014;44(5):928-33. https://doi.org/10.1007/s11430-013-4809-9 (in Chinese).

Li K, Gao J, Ju XD, Zhu J, Xiong Y, Liu S. Study on ultra-deep azimuthal electromagnetic resistivity LWD tool by influence quantification on azimuthal depth of investigation and real geosignal. Pure appl Geophys. 2018;175(12):4465-82. https://doi.org/10.1007/ s00024-018-1899-5.

Li Q, Omeragic D, Chou L, Yang L, Duong K. New directional electromagnetic tool for proactive geosteering and accurate formation evaluation while drilling. In: SPWLA 46th annual logging symposium, 26-29 June 2005, New Orleans, Louisiana; 2005.

Liu NZ, Wang Z, Liu C. Theories and key techniques of directional electromagnetic propagation resistivity tool for geosteering applications while drilling. Chin J Geophys. 2015;58(5):1767-75. https ://doi.org/10.6038/cjg20150526 (in Chinese).

Metropolis N, Rosenbluth AW, Rosenbluth MN, Teller AH, Teller E. Equation of state calculations by fast computing machines. $\mathrm{J}$ Chem Phys. 1953;21:1087-91. https://doi.org/10.1063/1.1699114.
Puzyrev V, Torres-Verdin C, Calo V. Interpretation of deep directional resistivity measurements acquired in high-angle and horizontal wells using 3-D inversion. Geophys J Int. 2018;213(2):1135-45. https://doi.org/10.1093/gji/ggy047.

Sambridge M. A parallel tempering algorithm for probabilistic sampling and multimodal optimization. Geophys J Int. 2014;196:35774. https://doi.org/10.1093/gji/ggt342.

Seydoux J, Legendre E, Mirto E, Dupuis C, Denichou JM, Bennett N, et al. Full 3D deep directional resistivity measurements optimize well placement and provide reservoir-scale imaging while drilling. In: SPWLA 55th annual logging symposium, 18-22 June 2014, Abu Dhabi, United Arab Emirates; 2014.

Wang HM, Shen QY, Chen JF. Sensitivity study and uncertainty quantification of azimuthal propagation resistivity measurements. In: SPWLA 59th Annual Logging Symposium, 2-6 June 2018, London, UK; 2018a.

Wang L, Fan YR, Huang R, Han YJ, Wu ZG, Xing DH, et al. Three dimensional Born geometrical factor of multi-component induction logging in anisotropic media. Acta Phys Sin. 2015;64(23):239301. https://doi.org/10.7498/aps.64.239301 (in Chinese).

Wang L, Li H, Fan YR, Wu Z. Sensitivity analysis and inversion processing of azimuthal resistivity logging-while-drilling measurements. J Geophys Eng. 2018b;15:2339-49. https://doi. org/10.1088/1742-2140/aacbf4.

Wang L, Fan YR, Yuan C, Wu Z, Deng S, Zhao W. Selection criteria and feasibility of the inversion model for azimuthal electromagnetic logging while drilling. Pet Explor Dev. 2018c;45(5):914-22. https://doi.org/10.1016/s1876-3804(18)30101-0 (in Chinese).

Wang L, Fan YR. Fast inversion of logging while drilling azimuthal resistivity measurements for geosteering and formation evaluation. J Petrol Sci Eng. 2019;176:342-51. https://doi.org/10.1016/j. petrol.2019.01.067.

Wang HS, Yang SW, Bai Y, Chen T, Wang HN. Three-dimensional finite volume simulation of the response of azimuth electromagnetic wave resistivity while drilling in inhomogeneous anisotropic formation. Acta Phys Sin. 2016;65(7):079101. https://doi. org/10.7498/aps.65.079101 (in Chinese).

Wei BJ, Tian K, Zhang X, Liu K. Physics of directional electromagnetic propagation measurements-while-drilling and its application for forecasting formation boundaries. Chin J Geophys. 2010;53(10):2507-15. https://doi.org/10.1002/cjg2.1558 (in Chinese).

Wu ZG, Fan YR, Wang L, Liu J, Xing T. Numerical modeling and analysis of eccentricity effect on borehole response of azimuthal electromagnetic logging-while-drilling tool. J China Univ Pet. 2017;41(5):69-79. https://doi.org/10.3969/j.issn.16735005.2017.05.008 (in Chinese).

Yang Z, Yang JZ, Han LJ, LI C. Interface detection performance analysis of azimuthal electromagnetic measurements while drilling. Acta Pet Sin. 2016;37(7):930-8. https://doi.org/10.7623/syxb2 01607012 (in Chinese).

Zeng S, Chen F, Li D, Chen J, Chen J. A novel 2.5D finite difference scheme for simulations of resistivity logging in anisotropic media. J Appl Geophys. 2018;150:144-52. https://doi.org/10.1016/j. jappgeo.2018.01.021. 